\title{
Future Results on Fragmentation Functions from BESIII
}

\author{
Yinghui Guan ${ }^{1}$, a , Isabella Garzia ${ }^{2}$, Haibo Li ${ }^{1}$, Xiao-Rui Lyu ${ }^{3}$, et Wenbiao Yan ${ }^{4}$ \\ ${ }^{1}$ Institute of High Energy Physics, CAS \\ ${ }^{2}$ Ferrara University, INFN \\ ${ }^{3}$ University of Chinese Academy of Sciences \\ ${ }^{4}$ University of Science and Technology of China
}

\begin{abstract}
Résumé. In this report, prospects on fragmentation function measurements at the BESIII experiment, including the inclusive production of hadrons and Collins effect measurements, are reported. These measurements are important to test the energy evolution effect of the fragmentation functions. Preliminary studies based on Monte Carlo simulations are presented.
\end{abstract}

\section{Introduction}

Fragmentation functions (FFs), which are denoted as $D_{h}^{i}(z)$, describe the probability that hadron $h$ carrying a fraction $z$ of parton's energy is found in the debris of the parton $i$. The universality of FFs is assumed in QCD regime, that is they are the same in different reaction process, thus can be measured from a limited set of reactions and used as input in other processes. Since the $D_{i}^{h}(z)$ incorporate the long distance non-perturbative physics of the hadronization processes, they can not be calculated from the QCD first principle but are usually parameterized with the aid of factorization and perturbative calculation. Then free parameters can be obtained by fitting to experimental data.

The unpolarized FFs has been studies for decades and a great success has been achieved. However, there is still a lack of data at low energy scale where the BESIII experiment just can contribute. On the other hand, transversespin-dependent effects in the fragmentation processes were first discussed by Collins [1] and the Collins fragmentation function was introduced. Measurement of FFs has great meaning to test our understanding of hadron processes. Besides, direct measurement of Collins function in $e^{+} e^{-}$collisions is very important to extract the transverse quark spin distributions in the nucleon (the socalled transversity) in semi-inclusive deep inelastic scattering (SIDIS) experiments. The first observation of the Collins effect in $e^{+} e^{-}$annihilations was reported by the Belle collaboration [2] and was updated based on a larger data sample at Belle later [3]. Collins effects measurements were also implemented at the BABAR experiment recently [4]. The combined analyses were performed to extract transversity using information from SIDIS and Belle data [5]. However, in this procedure, the energy evolution of FFs have to be considered because of the very

\footnotetext{
a. e-mail: guanyh@ihep.ac.cn
}

different energy scale of SIDIS and Belle (or BABAR). In fact, by now, the scale dependence of Collins FF is unknown and is assumed as same for the unpolarized FFs [5].

The BESIII, which is $e^{+} e^{-}$annihilation experiment, works at moderate energy scale, thus is a good playground to measure Collins FF for SIDIS and also can test the evolution effect of Collins function by comparing results from Belle and BABAR. This was proposed by Ref. [6], which gave prediction that the Collins effects at $4.6 \mathrm{GeV}$ will be larger than that measured at $10.6 \mathrm{GeV}$.

\section{BEPCII and BESIII}

The BEPCII [7] is a electron-positron symmetric collider with unpolarized beams, and it has $10^{33} \mathrm{~cm}^{-2} \mathrm{~s}^{-1} \mathrm{de}-$ signed luminosity. The beam energy is designed in the range $1.0-2.3 \mathrm{GeV}$.

The BESIII detector is a general symmetric purpose detector. It includes a Main Drift Chamber (MDC) for measuring momentum and specific ionization of charged particles, a Time of Flight (ToF) to aid particle identification and a CsI calorimeter (EMC) for detection of electromagnetic showers. These components are immersed in a magnetic field of $1 \mathrm{~T}$, provided by a superconducting solenoid, and surrounded by a multi-layer resistive plate counter system (MUC) to identify $\mu$ meson. More details on the features and capabilities of BESIII can be found elsewhere [8].

For the main physical purpose, most of data are taken at the center-of-mass (c.m.) energy of $3.01-4.6 \mathrm{GeV}$ at BESIII. While for the FFs study, a small off-resonances data set was used owing to clean resonance decay backgrounds, corresponding to the c.m. energy of $3.65 \mathrm{GeV}$ and a total luminosity of about $65 \mathrm{pb}^{-1}$. 

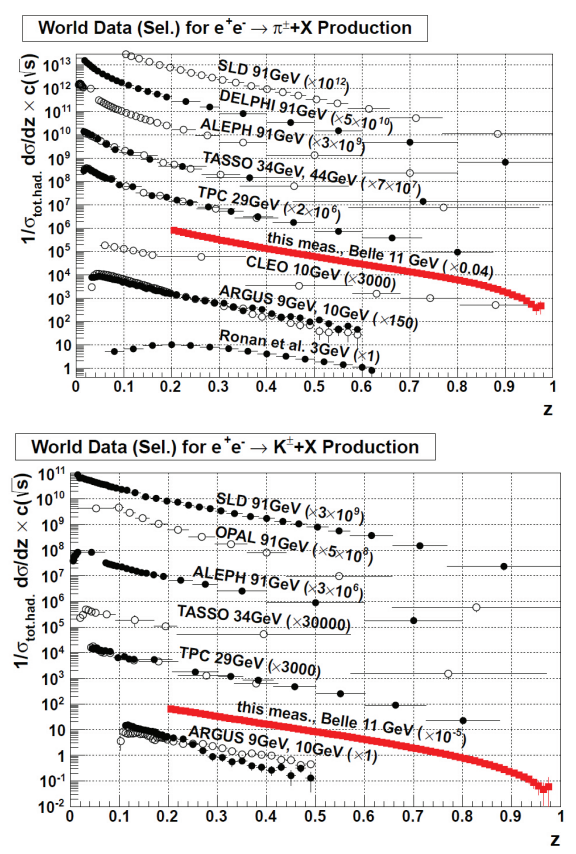

Figure 1. The newest charge-integrated normalized pion(up) and kaon(bottom) cross sections (multiplicities) from selected measurements [9].

\section{Perspectives on Fragmentation Functions}

\subsection{Inclusive production of $\pi, K$}

To test the unpolarized FFs, one is interested in the integrated cross section of the (charged and neutral) hadrons. There is a lack of information at the low energy scale [9] and high $z$ range, as shown in Fig. 1, where BESIII can contribute. While it is also a challenge for charged $\pi$ or $K$ with high fractional energy $z$, due to the high rate of the mis-identification of $\pi$ and $K$ with very high momentum. In contrast, studying on $\pi^{0}$ is a good opportunity because particle identification is not needed. Especially, EMC which measures the neutral tracks at BESIII has good performance. Neutral kaon, $K_{S}$, is also a clean channel because of the flight distance of the $K_{S}$, thus its decay vertex is very useful to suppress backgrounds. Measurements of the inclusive production of $K_{S}$ now is in progress at BESIII. Preliminary Monte Carlo (MC) simulations shows that the backgrounds are very few and the resolution of the $K_{S}$ momentum, which is also crucial to study the $z$ dependence of the FFs, is good enough.

\subsection{Collins asymmetries in inclusive charged pions}

\subsubsection{Cross section}

The Collins fragmentation function describes the production of a hadron with transverse momentum $\mathbf{P}_{h \perp}$ from a transversely polarized quark with spin $\mathbf{S}_{\mathbf{q}}$ and momentum

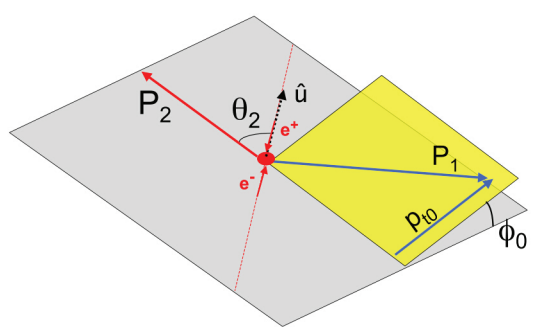

Figure 2. The definition of azimuthal angle $\phi_{0}$ in the second hadron frame [10].

k. The number densities for finding a hadron $h$ produced from a transversely polarized quark $q$ can be defined as :

$$
D_{h, q \uparrow}=D_{1}^{q}\left(z, P_{h \perp}^{2}\right)+H_{1}^{\perp q}\left(z, P_{h \perp}^{2}\right) \frac{\left(\hat{\mathbf{k}} \times \mathbf{P}_{\mathbf{h} \perp}\right) \cdot \mathbf{S}_{\mathbf{q}}}{z M_{h}} .
$$

The first term is the unpolarized fragmentation function, while the second term contains the Collins fragmentation function $H_{1}^{\perp q}\left(z, P_{h \perp}^{2}\right)$ and the spin orientation of the quark. The Collins function describes the relationship between the quark spin and the creation of final hadrons. The vector product introduces a $\cos (\phi)$ modulation where $\phi$ is the azimuthal angle spanned by the transverse momentum and the plane normal to the quark spin along the quark's momentum. The modulation is the so-called single spin asymmetries (SSA).

Same as Belle [3] and BABAR [4], with the unpolarized beams, the SSA caused by a single Collins function can not be observed at BESIII. Instead, the Double Collins Asymmetries (DCA) can be explored by looking at the two correlated Collins functions simultaneously. Specifically, in $e^{+} e^{-} \rightarrow q \bar{q}$ process, due to the spin of the virtual photon, the spin direction of the $q$ and $\bar{q}$ must be parallel. This is the correlation between the Collins FFs of the quark and the anti-quark. To probe DCA, the azimuthal angles can be defined in two frames : the jet reference frame and the second hadron frame [10]. However, only the later one was introduce at BESIII, because it is too hard to define jets in the case of the low energy and low multiplicity. In the second hadron frame, the azimuthal angle $\phi_{0}$ is defined as the angle between the plane spanned by the beam axis and the second hadron $\mathbf{P}_{\mathbf{2}}$, and the transverse momentum $\mathbf{p}_{\mathbf{t} 0}$ of the first hadron around the second hadron direction, as shown in Fig. 2. And the cross section follows the expression

$$
\sigma\left(e^{+} e^{-} \rightarrow h_{1} h_{2} X\right) \propto \cos \left(2 \phi_{0}\right) H_{1}^{\perp}\left(z_{1}\right) \otimes H_{2}^{\perp}\left(z_{2}\right),
$$

where two Collins FFs are included and a $\cos \left(2 \phi_{0}\right)$ modulation is introduced. In the ongoing analysis at BESIII, only the inclusive charged $\pi$ is studied now.

\subsubsection{Double Ratio}

Referring to Belle and BABAR's measurements, double ratio are constructed to cancel detector effects and 
QCD radiative effects to the first order. First, the normalized distribution of $2 \phi_{0}$ is defined as

$$
R_{0}:=\frac{N\left(2 \phi_{0}\right)}{\left\langle N_{0}\right\rangle}
$$

where the $\left\langle N_{0}\right\rangle$ is the average bin content. The normalized ratio are built for unlike-sign $\left(\pi^{ \pm} \pi^{\mp}\right)$, like-sign $\left(\pi^{ \pm} \pi^{ \pm}\right)$ and charged pion-pairs $(\pi \pi)$, respectively. Then the double ratio of unlike-sign pair $\left(R^{U}\right)$ over like-sign pair $\left(R^{L}\right)$ are built as $R^{U} / R^{L}$, which can be expressed as

$$
\frac{R^{U}}{R^{L}} \propto 1+\frac{\sin _{\theta_{2}}^{2}}{1+\cos ^{2} \theta_{2}} \cos \left(2 \phi_{0}\right) \mathcal{F}\left(\frac{H_{1}^{\perp}\left(z_{1}\right) \bar{H}_{1}^{\perp}\left(z_{2}\right)}{D_{1}\left(z_{1}\right) \bar{D}_{1} z_{2}}\right),
$$

The $\cos \left(2 \phi_{0}\right)$ modulation still exist, while detection effects which are charge-independent, can be cancelled out. This is because that the unlike-sign and like-sign $\pi$ pairs production involve different combination of favored FFs and disfavored FFs which are charge dependent, thus are not cancelled in double ratio. Details can be found in Ref. [3, 4]. Similarly, the double ratio of $R^{U}$ over $R^{C}$ can be built. Fit to the double ratio using the following function

$$
\frac{R^{U}}{R^{L(C)}}=A \cos \left(2 \phi_{0}\right)+B
$$

where $A$ and $B$ are free parameters, and $A_{U L(C)}=\frac{B}{A}$ denotes the Collins asymmetries of interest.

\subsubsection{Detection effects}

Different kinds of detector effects are found and carefully checked in our studies. First, charged pion pairs with same charge are found having relatively lower reconstruction efficiencies than that with opposite charge when $\phi_{\mathrm{Lab}}$ of the two pions are close, where $\phi_{\text {Lab }}$ denotes the azimuthal angle of a track in the lab frame. In this case, same sign tracks share more MDC wires in the detector, especially when their transverse momentum is close and thus their curvity are almost equivalent, as illustrated in Fig. 3. The second detection effect comes from the fake tracks companying with nearby real tracks, the so-called "ghost tracks", which are also caused by unperfect tracks finding procedure. In this case, fake like-sign hadron pair are created and usually they have very small open angle. These detector effects cause the different efficiencies of unlikesign and like-sign pairs, thus can not be cancelled in double ratio, but can be suppressed by requiring large open angle of the two pions.

\subsubsection{MC validation}

Preliminary studies on measuring Collins FF are performed based on the MC sample. For the promising data set taken at continuum region, the background level is expected to be very low and this is verified by MC. The main backgrounds $e^{+} e^{-} \rightarrow \tau^{+} \tau^{-}$process has the fraction of about only $2 \%$ and other backgrounds are negligible. The performance of detector and the resultant $2 \phi_{0}$ distribution are investigated using pure $e^{+} e^{-} \rightarrow q \bar{q}(q=u d s)$

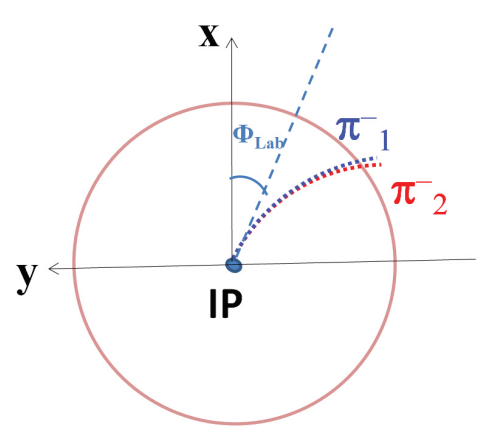

Figure 3. Inefficiencies of like-sign charged hadron, due to the share of MDC wires.

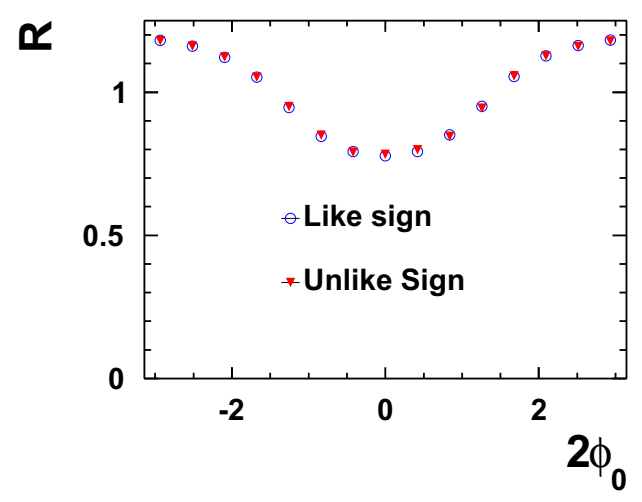

Figure 4. The normalized ratio of unlike-sign and like-sign $\pi$ pairs in MC.

MC sample. The normalized distributions of $2 \phi_{0}$ of unlikesign and like-sign pairs are shown in Fig. 4. To study the $z$-dependence of Collins asymmetries, double ratio of $2 \phi_{0}$ are studies in different $z$ region. To estimate the sensitivity of data, the equivalent MC sample is used. The obtained $A_{U L}$ in MC are shown in Fig. 5, with the statistical error bar shown. With the prediction of large Collins asymmetries in the high $z$ region, asymmetries are expected to be visible in the last $z$ bin. Conservatively, specific sensitivity are not given in this report. It is relevant to our understanding of the issue of "mis-combination rate", which will be discussed in the next section.

\subsubsection{Mis-combination of hadrons}

Although, mature techniques and analysis strategy can be learned from Belle and BABAR, one has to realize the very different situation at BESIII. One important thing is that, at BESIII, it is almost impossible to separate hadrons from $q$ and $\bar{q}$ due to the absence of jets. While all hadronpairs are taken account, two hadrons come from the same quark are also combined as pairs and included. These combination (from same parton) are not expected to generate any azimuthal asymmetries but just contribution to the normalized factor, and as a consequence, dilute the asymmetries of interest. The measured asymmetries should be cor- 


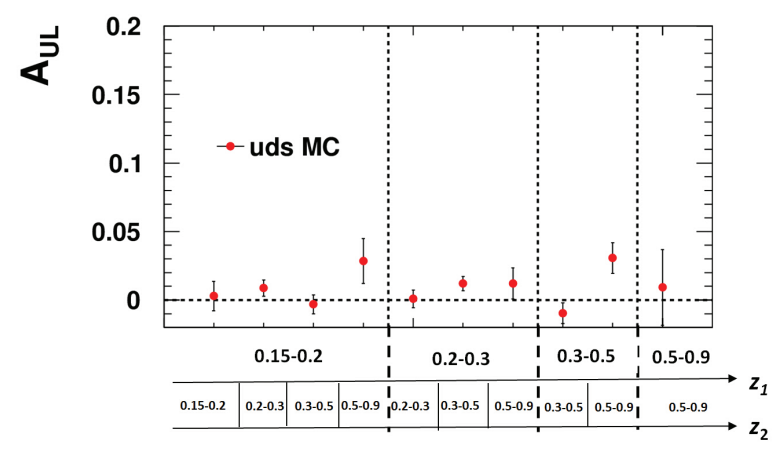

Figure 5. The $A_{U L}$ obtained in $u d s$ MC. Symmetrized $z$-bin subdivisions are used, where, for instance, the bin of " $z_{1} \in[0.15,0.20]$, $z_{2} \in[0.20,0.30]$ " includes all $\pi$ pairs with one pion has fraction energy in the range of $[0.15,0.2]$ and the other is in the range of $[0.20,0.30]$.

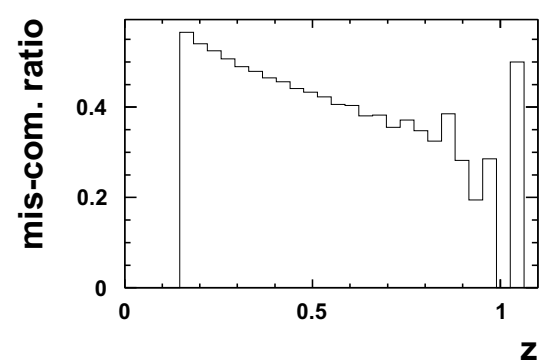

Figure 6. The rate of mis-combination, $R_{\text {mis }}$, which are obtained from MC.

rected according the following equation

$$
A^{\text {true }} \approx A^{\text {mea. }}\left(1+R_{\text {mis }}\right) \text {, }
$$

where the $R_{\text {mis }}$ stands for the rate of mis-combination and need to be known. In our analysis, in order to obtain $R_{\text {mis }}$, the final hadrons were traced back to the initial quark (antiquark) in the MC simulation with Pythia [11] generator.
The obtained $R_{\text {mis }}$ are shown in Fig. 6, one can see that the $R_{\text {mis }}$ is slightly high at BESIII energy scale. In addition, the $R_{\text {mis }}$ obtained from MC is model-dependent, other methods are under study to estimate $R_{\text {mis }}$.

\section{Summary and outlook}

In summary, we reported the plans and progress on fragmentation functions measurements at BESIII. The BESIII works at moderate energy scale, which is an ideal playground to test the energy evolution effects of FFs. Both the unpolarized and polarized FFs are of interest, among which inclusive production of $K_{S}$ and Double Collins Asymmetries measurements are in progress with a small off-resonance data sample. The Collins effect measurement may be extended to the large data sample above 4.0 GeV, however, which requires a careful investigation of the background contributions.

\section{Références}

[1] J. C. Collins, Nucl. Phys B396, 161 (1993).

[2] R. Seidl et al. (Belle Collaboration), Phys. Rev. Lett 96, 232002 (2006).

[3] R. Seidl et al. (Belle Collaboration), Phys. Rev. D 78, 032011 (2008).

[4] Lees, J. P. et al. (BaBar Collaboration), Phys, Rev. D 90, 052003 (2014).

[5] M. Anselmino et al., Phys. Rev. D 87, 094019 (2013).

[6] P. Sun, F. Yuan, Phys. Rev. D 88, 034016 (2013).

[7] C. Zhang, Sci. China G 53, 2084 (2010).

[8] M. Ablikim et al. (BESIII Collaboration), Nucl. Instrum. Meth. A614, 345 (2010).

[9] Leitgab, M et al. (Belle Collaboration), Phys. Rev. Lett 111, 062002 (2013).

[10] D. Boer, R. Jakob and P.J. Mulders, Nucl. Phys. B 504, 345 (1997).

[11] T. SjÖstrand, S. Mrenna and P. Skands, JHEP 0605, 026 (2006). 